\title{
Biomarkers for neonatal therapeutic studies: time for a public-private partnership
}

\author{
Scott C. Denne ${ }^{1}$, Jonathan M. Davis ${ }^{2}$ and on behalf of the Pediatric Policy Council
}

$E^{n}$ nsuring that children have access to safe and effective drugs has been a concern of pediatricians for over 40 years. In 1977, the American Academy of Pediatrics Committee on Drugs formally called for drugs to be tested in children. After 25 years of incremental regulatory improvements, the Best Pharmaceuticals for Children Act (BPCA) was signed into law in 2002. The BPCA encouraged drug studies in children by granting an additional 6 months of patent exclusivity if pediatric studies were conducted. While this "carrot" approach had some success, it was supplemented by the Pediatric Research Equity Act (PREA) in 2003, which mandates the study of drugs or biologics if those products are likely to be useful for children. These acts were initially subject to periodic congressional reauthorization, but in 2012 both BPCA and PREA were made permanent. These regulatory changes have resulted in significant improvements with over 600 new studies conducted in children to support pediatric drug-labeling information.

Despite these successes, significant challenges remain for enhancing pediatric drug development including: (1) relatively small numbers of children are eligible for clinical trials (since much of pediatrics involves rare diseases), (2) ensuring appropriate safety measures, and (3) identifying achievable clinical outcomes. These obstacles are particularly problematic for the neonatal population with drug studies in neonates lagging behind studies in older children. There is an urgent need for alternative methods and approaches that can increase the number, speed, and quality of neonatal drug studies.

Well-characterized, validated biomarkers hold significant promise to accelerate neonatal drug development and testing. Although by no means a magic bullet, biomarkers may be able to facilitate more efficient monitoring for drug safety, or serve as surrogate endpoints. In this issue of Pediatric Research, Kelly et al. (1) evaluated the quality of response biomarker reporting in neonatal clinical trials. They identified huge variability in the selection, measurement, and reporting of neonatal response biomarkers in prospective interventional studies, limiting their usefulness for assessments of safety or efficacy. At present, there is no systematic organized approach within the pharmaceutical industry, government agencies, or the academic community to develop, test, and validate neonatal biomarkers for clinical drug trials.

Fortunately, there is great interest from federal agencies and foundations in developing biomarkers for therapeutic studies, especially in neonates. For example, the National Institutes of Health (NIH) has an open funding opportunity focused on testing biomarkers in the pediatric population that have been validated in adults (2). The NIH and FDA created a joint biomarker working group that recently produced a valuable biomarker resource document (3). To promote biomarker development, the FDA has implemented a biomarker qualification program; submission of biomarkers can come from a variety of stakeholders including academia, consortia, disease foundations, and patient advocacy groups (4). Finally, to encourage multiple stakeholders to work together, a Biomarkers Consortium was established. This public-private biomedical research partnership is managed by the Foundation for the NIH (5). The Consortium focuses on identifying, developing, and achieving regulatory approval for biomarkers to support new drug development and medical diagnostics. At present, there are over 25 distinct biomarker consortium programs focused on particular diseases or populations. Currently, none of these consortia include neonates.

It is time for a robust, organized partnership of academia, industry, and government to focus on the development, testing, and validation of neonatal biomarkers. The NIH and FDA can strongly encourage testing of one or more biomarkers in all Phase III clinical trials of neonates. There is great potential for these efforts that begin to address the substantial barriers to the development of new and existing drugs for neonates. With over $\$ 30$ billion per year spent on the treatment of prematurity, it is critical that we work together to better ensure that safe and effective treatments are readily available for this highly vulnerable population. Consortia such as the International Neonatal Consortium are in an excellent position to play an important role going forward (6).

\footnotetext{
${ }^{1}$ Department of Pediatrics, Indiana University, Indianapolis, Indiana; ${ }^{2}$ Department of Pediatrics and the Tufts Clinical and Translational Research Institute, Tufts University, Boston, Massachusetts.

Correspondence: S.C. Denne (sdenne@iu.edu)

Received 12 October 2017; accepted 16 October 2017; advance online publication 22 November 2017. doi:10.1038/pr.2017.281
} 


\section{Commentary | Denne et al.}

\section{STATEMENT OF FINANCIAL SUPPORT}

No financial support was received for this commentary.

Disclosure: The authors declare no conflict of interest.

\section{REFERENCES}

1. Kelly L, Caswell K, Short M, Parimi PS, Offringa M, Diacovo T. Response biomarkers in neonatal intervention studies. Pediatric Research (in press).

2. National Institutes of Health Funding Opportunity: Biomarkers: Bridging Pediatric and Adult Therapeutics. https:/grants.nih.gov/grants/guide/pafiles/PAR-17-169.html.
3. National Institutes of Health-Food and Drug Administration Working Group: BEST (Biomarkers, EndpointS and other Tools) Resource. https:// www.ncbi.nlm.nih.gov/books/NBK326791/.

4. Food and Drug Administration Biomarker Qualification program. https://www.fda.gov/Drugs/DevelopmentApprovalProcess/DrugDevelopment ToolsQualificationProgram/BiomarkerQualificationProgram/ucm 20086360. htm.

5. Foundation for the National Institutes of Health Biomarkers Consortium. https://fnih.org/what-we-do/biomarkers-consortium.

6. Davis JM, Turner MA. Global collaboration is needed to develop new and existing drugs for neonates. JAMA Pediatr 2015; 169:887-8. 